UCRHEP-T354

ULB-TH/03-16

NSF-KITP-03-32

May 2003

\title{
Gaugino-induced quark and lepton masses in a truly minimal left-right model
}

\author{
J.-M. Frère ${ }^{1,3}$ and Ernest $\mathbf{M a}^{2,3}$ \\ 1 Service de Physique Théorique, Université Libre de Bruxelles, B-1050 Brussels, Belgium \\ 2 Physics Department, University of California, Riverside, California 92521, USA \\ ${ }^{3}$ Kavli Institute for Theoretical Physics, University of California, \\ Santa Barbara, California 93106, USA
}

\begin{abstract}
It has recently been proposed that all fundamental fermion masses (whether Dirac or Majorana) come from effective dimension-five operators in the context of a truly minimal left-right model. We show how a particularly economical scheme emerges in a supersymmetric framework, where chiral symmetry breaking originates in the gaugino sector.
\end{abstract}


In the Standard Model of particle interactions, the spontaneous breaking of the $S U(2)_{L} \times$ $U(1)_{Y}$ gauge symmetry to $U(1)_{e m}$ is achieved through the vacuum expectation value of the scalar doublet $\Phi=\left(\phi^{+}, \phi^{0}\right)$. At the same time, since left-handed quarks and leptons are doublets under $S U(2)_{L} \times U(1)_{Y}$ whereas right-handed quarks and leptons are singlets, chiral symmetry is also broken by $\left\langle\phi^{0}\right\rangle$, thus allowing quarks and leptons to acquire the usual Dirac masses. The only exception is the neutrino which gets a small Majorana mass through the unique dimension-five operator [1, 2]

$$
\mathcal{L}_{\Lambda}=\frac{f_{i j}}{2 \Lambda}\left(\nu_{i} \phi^{0}-e_{i} \phi^{+}\right)\left(\nu_{j} \phi^{0}-e_{j} \phi^{+}\right)+H . c .
$$

Suppose we now extend the standard-model gauge symmetry to $S U(3)_{C} \times S U(2)_{L} \times$ $S U(2)_{R} \times U(1)_{B-L}[3]$, then the spontaneous breaking of $S U(2)_{R} \times U(1)_{B-L}$ to $U(1)_{Y}$ is simply achieved by the scalar doublet

$$
\Phi_{R}=\left(\phi_{R}^{+}, \phi_{R}^{0}\right) \sim(1,1,2,1)
$$

where the notation refers to the dimension of the non-Abelian representation or the value of the Abelian charge $B-L$ or $Y$ in the convention

$$
Q=I_{3 L}+I_{3 R}+\frac{1}{2}(B-L)=I_{3 L}+\frac{Y}{2}
$$

while the corresponding field

$$
\Phi_{L}=\left(\phi_{L}^{+}, \phi_{L}^{0}\right) \sim(1,2,1,1)
$$

becomes the same as the usual scalar doublet of the Standard Model, and breaks $S U(2)_{L} \times$ $U(1)_{Y}$ in turn to $U(1)_{e m}$.

In other words, $\Phi_{R}$ and $\Phi_{L}$ are sufficient by themselves for the desired breaking of the left-right gauge symmetry all the way down to $U(1)_{\mathrm{em}}$. On the other hand, chiral symmetry of the quarks and leptons remains unbroken at this stage, in contrast to the case of the 
Standard Model. The conventional way to deal with this problem in the left-right context is to introduce a "bidoublet" $(1,2,2,0)$ which, combined with either $\Phi_{R}$ or a similar triplet, generates both gauge-boson and fermion masses. It is however also well-known that this would result in the existence of flavour changing neutral currents (FCNC), which must be controlled in some way. This solution is also particularly unpleasant if one extends the model to include supersymmetry. In that case, two bidoublets (instead of one) must be introduced (the equivalent of 4 standard doublets) and the problem with FCNC is compounded.

Suppose we do not introduce this scalar bidoublet, then fermion masses are still possible, but they (whether Dirac or Majorana) must all come from dimension-five operators, as recently proposed [4]. We would then have the advantage of a uniform understanding of the origin of all fundamental fermion masses.

The obvious (and first) proposal [5] to generate such operators, outside of supersymmetry, is to introduce one massive vectorlike $S U(2)_{L} \times S U(2)_{R}$ singlet fermion per observed lowenergy fermion species. While this approach might be otherwise minimal, an easier and more economical mechanism exists if we extend the model to include supersymmetry.

Before working out the details, suppose we simply consider the supersymmetric extension of the scheme above, then again the quarks and leptons are massless without the dimensionfive operators. However, we now have the very interesting option of obtaining these operators radiatively through a special class of supersymmetry breaking terms, as was done for the left-handed neutrino Majorana mass in Ref. [7. The resulting quark and lepton masses are then derived as functions of the squark and slepton masses together with those of the gauginos [8, 9].

We now turn to the minimal field content of such a model. Under $S U(3)_{C} \times S U(2)_{L} \times$ $S U(2)_{R} \times U(1)_{B-L}$, the quark and lepton superfields transform as

$$
q \sim(3,2,1,1 / 3), \quad q^{c} \sim\left(3^{*}, 1,2,-1 / 3\right)
$$




$$
l \sim(1,2,1,-1), \quad l^{c} \sim(1,1,2,1)
$$

where we have adopted the convention that all fermion fields are left-handed, with their righthanded counterparts denoted by the corresponding (left-handed) charge-conjugate fields. Thus we have used here for the left-handed superfield $q^{c}$ the implicit notation $q^{c}=\left(q_{R}\right)^{c} \neq$ $\left(q_{L}\right)^{c}$. We add the following Higgs superfields:

$$
\begin{aligned}
\Phi_{L} \sim(1,2,1,1), & \Phi_{R} \sim(1,1,2,-1), \\
\Phi_{L}^{c} \sim(1,2,1,-1), & \Phi_{R}^{c} \sim(1,1,2,1),
\end{aligned}
$$

These fields allow for the obvious bilinear terms $\left(\Phi_{L} \Phi_{L}^{c}\right)$ and $\left(\Phi_{R} \Phi_{R}^{c}\right)$, but also the unwanted lepton-number violating terms $\left(l_{L} \Phi_{L}\right)$ and $\left(l^{c} \Phi_{R}\right)$, which we must forbid by an unbroken discrete symmetry, i.e. all quark and lepton superfields are odd, but all Higgs superfields are even. In terms of their components, $(q \Phi)$ is a short-hand notation for $\epsilon_{i j} q_{i} \phi_{j}$, etc.

Since we do not admit Higgs bidoublets, there are no trilinear terms in the superpotential of this model. This means that all quarks and leptons remain massless even though $S U(3)_{C} \times$ $S U(2)_{L} \times S U(2)_{R} \times U(1)_{B-L}$ has been broken down to $S U(3)_{C} \times U(1)_{e m}$. In other words, chiral symmetry breaking has now been separated from gauge symmetry breaking.

Before discussing our main result, namely the generation of light fermion masses, we want to point out that the scalar structure of our model at low energy is identical to that of the Minimal Supersymmetric Standard Model (MSSM), once the $S U(2)_{R}$ fields get large masses and/or vacuum expectation values. The specific FCNC problems of nonminimal left-right models are thus avoided. On the other hand, FCNC could still be induced by the flavour structure of the supersymmetry breaking sector.

To generate quark and lepton masses, we must add dimension-five operators as proposed in Ref. 4, i.e. $\left(q \Phi_{L}\right)\left(q^{c} \Phi_{R}\right)$ for $m_{u}$ and $\left(q \Phi_{L}^{c}\right)\left(q^{c} \Phi_{R}^{c}\right)$ for $m_{d}$, etc. As already stated, we can avoid here the extra fields required by the "universal seesaw" mechanism [5], where each 
quark or lepton has a heavy singlet counterpart. In a supersymmetric model, no such fields are needed. Instead, light fermion masses may be generated by taking into account the presence of "nonstandard" (but usually present) supersymmetry breaking terms, as shown below.

Such terms are NOT a new addition. In most phenomenological models, supersymmetry is broken in a more or less "hidden" sector, and characterized by the vacuum expectation value of some (auxiliary) field $F$. This hidden-sector breaking is then transferred to "our" world by some mediator (possibly gravity itself), to which we associate a mass scale $M$. This results in a classification of "effective" supersymmetry breaking terms, obtained by an expansion in $F / M \approx m_{\text {susy }}$ where $m_{\text {susy }}$ is adjusted to be the mass gap between the known particles and their superpartners, which we may want to keep in the 1 to $10 \mathrm{TeV}$ range to maintain the stability of the electroweak scale.

This expansion is usually kept to lowest significant order in $1 / M$, which yields "superrenormalisable" (or "soft") terms, like sfermion masses, trilinear scalar couplings or gaugino masses. There is no reason however not to continue with such an expansion, other than the expected smallness of the effects. Of course, since the expansion parameter is dimensional, this will generate "hard" couplings. It should however be of little concern, since their impact will be small in general, unless the integration over such terms reaches the scale $M$, where the full theory acts as a natural cutoff.

An extensive study and classification of such terms is found in Ref. [6], with the conclusion that beyond the usual soft breakings, the first corrections are of order $F / M^{2}$ and introduce operators of the type $\phi \lambda \lambda$ and $\phi^{4}$ (the latter to be distinguished from $\phi^{* 2} \phi^{2}$, which only appears at order $F^{2} / M^{4}$ ). (Here $\lambda$ represents a gaugino and $\phi$ the scalar part of a chiral superfield). We are interested now in the quartic $\phi^{4}$ term, which was actually already used in a more restricted context in Ref. [7]. There, the dimension-four supersymmetry breaking 
term $h\left(\epsilon_{i j} \tilde{l}_{i} \phi_{L, j}\right)^{2}$, where $\tilde{l}$ and $\phi_{L}$ are the scalar partners of the corresponding superfields, is used to generate a small Majorana meutrino mass. An example of such a contribution is given in Fig. 1. (For details, see Ref.[7]).

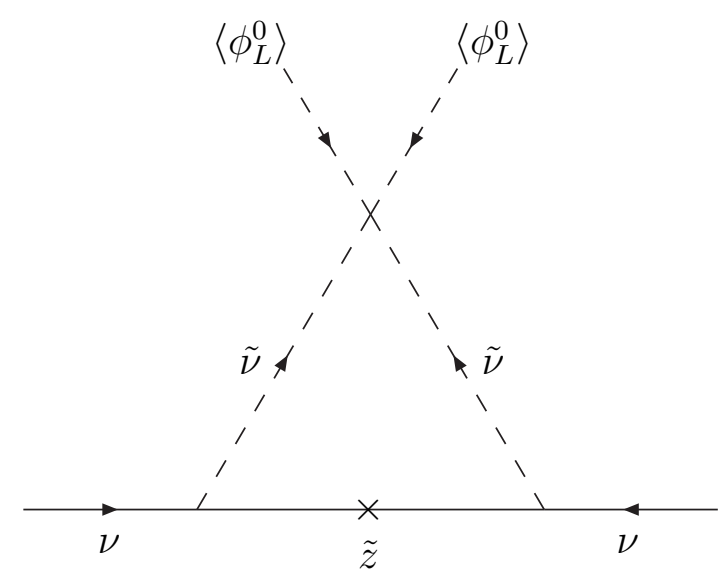

Figure 1: Example of a one-loop generation of Majorana neutrino mass.

This graph shows clearly how we differ from the canonical seesaw mechanism. The quantum numbers of the external fermions are carried through the graph by the sfermions (eliminating the need for heavy Majorana particles or heavy vectorlike partners), while the chiral symmetry breaking is provided in a (largely) universal way by the gauginos. Of course, the effect initially evaluated for left-handed neutrinos is tiny (as desired), but in the present left-right extension with operators such as $h\left(\tilde{q} \Phi_{L}\right)\left(\tilde{q}^{c} \Phi_{R}\right)$, etc., a Dirac mass would be enhanced by the large ratio of $\left\langle\phi_{R}^{0}\right\rangle=v_{R} / \sqrt{2}$ to $\left\langle\phi_{L}^{0}\right\rangle=v_{L} / \sqrt{2}$., implying that $S U(2)_{R}$ should be broken at a very high scale.

For the charged leptons, the graphs to be considered are similar to Fig. 1, but with both $S U(2)_{L}$ and $S U(2)_{R}$ fields involved, and the chirality flip is due again to the exchange of a neutralino (typically the partner of the light $U(1)_{Y}$ gauge field, usually named the bino $(\tilde{B})$, the only neutral gaugino to have both significant $S U(2)_{L}$ and $S U(2)_{R}$ couplings). In the case of quarks, both neutralino and gluino exchange must be considered, the latter providing 
the dominant contribution [8, 9], as shown in Fig. 2.

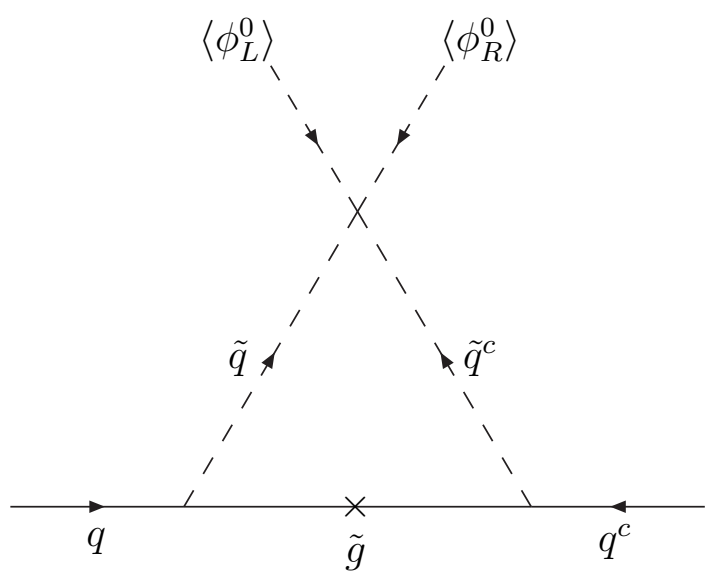

Figure 2: One-loop generation of quark mass.

The new term $v_{L} v_{R}$ induces mixing between $S U(2)_{L}$ and $S U(2)_{R}$ sfermions, and we should in principle diagonalize this matrix and deal with the exchanges of both eigenstates (see for instance Ref. 9]). However, excluding the case of $m_{t}$ (to be discussed later), the mixing term is always safely small, and the evaluation of the triangle graph is accurate enough. In the following we assume that all superpartner masses (gauginos, squarks, and sleptons) are similar and denoted by $\tilde{m}$, whereas $v_{L}$ refers to either of the 2 scalar doublet vacuum expectation values of the MSSM. We obtain in this way for the gluino contribution to the quark mass the order of magnitude

$$
m_{q} \simeq \frac{1}{32 \pi^{2}}\left(\frac{4}{3}\right)\left(\frac{2 g_{s}^{2}}{\tilde{m}}\right)\left(\frac{h v_{L} v_{R}}{2}\right)
$$

where we have used the quartic coupling $h\left(\tilde{q} \Phi_{L}\right)\left(\tilde{q}^{c} \Phi_{R}\right)$. Recalling our previous discussion on the origin of $h$, we set it equal to $\kappa \tilde{m} / M$ with $M$ the "mediator" mass and obtain

$$
m_{q} \simeq \frac{\alpha_{s} \kappa v_{L}}{6 \pi}\left(\frac{v_{R}}{M}\right)
$$

Note that the scale $\tilde{m}$ disappears from this equation. However there are indirect constraints which we must consider. Nevertheless, let us ignore these constraints for the moment and 
proceed to determine the necessary conditions for the radiative generation of realistic quark masses, the strongest requirement obviously coming from the top quark. Since $\kappa$ is expected to be of order unity or less, it is readily seen that realistic quark (and lepton) masses require a large $v_{R}$. For the top quark mass, $v_{R} / M \sim 10^{2}$ would be required. In "gravity-mediated" schemes, $M$ is already of order the Planck mass, which tells us that $v_{R}>M$ is not a reasonable choice. On the other hand, if $M$ is smaller, as in gauge-mediated supersymmetry breaking, $v_{R}>M$ is possible, but then $v_{R}>\sqrt{F}$ as well. In other words, $S U(2)_{R}$ breaking must occur without supersymmetry breaking. For example, if we choose $v_{R} \sim 10^{12} \mathrm{GeV}$ for leptogenesis, then $M \sim 10^{10} \mathrm{GeV}$ and $\sqrt{F} \sim 10^{7} \mathrm{GeV}$ for $m_{\text {susy }} \sim 10 \mathrm{TeV}$. At the same time, using the analog of Eq. (10), we obtain $m_{\nu} \sim 0.1 \mathrm{eV}$, which is just right for neutrino oscillations.

The large $\left(v_{R} / M\right)$ ratio also raises a strong concern that the expansion in powers of $1 / M$ may not be valid. This would certainly be true unless the connection between $v_{R}$ and the low-energy sector is systematically suppressed, such that the true expansion parameter is not $v_{R} / M$ but a smaller combination, as for example in the graph studied here. Whether or not this is actually realized depends on the (unknown) specific supersymmetry breaking mechanism. Imposing this suppression of the coupling has immediate consequences. Maintaining the supersymmetry mass scale $\tilde{m}$ in a reasonable range (say 1 to $10 \mathrm{TeV}$ ) requires that a large value of $v_{R}$ does not generate excessive off-diagonal corrections to the diagonal squark masses. This contribution is of course also given by the vertex already considered, and is of order $\kappa\left(v_{L} / \tilde{m}\right)\left(v_{R} / M\right)(\tilde{m})^{2}$. To keep this contribution of order $(\tilde{m})^{2}$, we require $\tilde{m} \sim v_{L}\left(v_{R} / M\right)$ and thus a rather large supersymmetry mass scale (of order $10 \mathrm{TeV}$ ). A potentially even stronger constraint comes from the quartic coupling $\left(\tilde{q}^{c} \Phi_{R}\right)\left(\tilde{q}^{c} \Phi_{R}\right)^{\dagger}$. Such a term is generically of order $F^{2} / M^{4}$ (see Ref. [6]) and generates a correction of order $(F / M)^{2}\left(v_{R} / M\right)^{2} \sim \tilde{m}^{2}\left(v_{R} / M\right)^{2}$. Keeping this correction under control with a large value 
of $v_{R} / M$ implies that the coefficient of this term is for some reason strongly suppressed or perhaps even absent.

If instead $v_{R} \sim M$ is assumed, all quark and lepton masses may still be obtained from Eq. (10) except for $m_{t}$. In that case, we may invoke the dynamical mechanism of Bardeen, Hill, and Lindner (BHL) [10] which also uses a higher-dimensional operator. [In the case of supersymmetry, this mechanism has also been discussed [11.] Since there are already fundamental scalars in supersymmetry which can share the vacuum expectation values of electroweak symmetry breaking, the correct $m_{t}$ from the BHL mechanism may also be obtained. [In the Standard Model, the simplest version of the BHL model predicts $m_{t}=226$ $\mathrm{GeV}$ for a cutoff of $10^{16} \mathrm{GeV}$ and even a bigger value for a lower cutoff.] As far as the low-energy phenomenology is concerned (at energies up to the supersymmetry scale $\tilde{m}$ ), the effective Yukawa couplings between light fermions and physical scalar bosons are then modified with respect to the MSSM, because there would be an additional effective BHL Higgs doublet.

We should also point out that in the present scheme, the chiral symmetry breaking can be traced first to the gaugino sector, and through it and the mediators, to the supersymmetry breaking scenario itself. In such circumstances, one might expect some form of "superlight axion mechanism" to take place, associating the axion primarily to the first source of chiral symmetry breaking, i.e. the dynamical phase of the gluino as proposed in Ref. [12]. The details depend of course on the choice of a definite model for the supersymmetry breaking mechanism.

In conclusion, we have proposed a supersymmetric left-right model, where the gauge symmetry breaking is achieved simply by 4 doublet superfields, without breaking the chiral symmetry of the quarks and leptons. The latter is accomplished instead by supersymmetry breaking terms involving gauginos, sfermions, and a quartic scalar interaction. We have thus 
a uniform understanding of all fermion masses (except possibly for $m_{t}$ ): they are radiatively generated by supersymmetry breaking and Dirac masses are much larger than Majorana masses because $v_{R}>>v_{L}$.

This work was supported in part by the U. S. Department of Energy under Grant No. DEFG03-94ER40837, by the French Community of Belgium under contracts IISN and ARC, by the Belgian federal government under IAP 5/27, and by the U. S. National Science Foundation under Grant No. PHY99-07949. In this last respect, the authors thank the KITP (U. C. Santa Barbara) for its gracious hospitality and friendly atmosphere, and the organisers of its 2003 Neutrino Workshop, which inspired this work. 


\section{References}

[1] S. Weinberg, Phys. Rev. Lett. 43, 1566 (1979).

[2] E. Ma, Phys. Rev. Lett. 81, 1171 (1998).

[3] J. C. Pati and A. Salam, Phys. Rev. D10, 275 (1974).

[4] B. Brahmachari, E. Ma, and U. Sarkar, Phys. Rev. Lett. 91, 011801 (2003).

[5] A. Davidson and K. C. Wali, Phys. Rev. Lett. 59, 393, (1987); S. Rajpoot, Phys. Rev. D36, 1479 (1987).

[6] S. P. Martin, Phys. Rev. D61 035004 (2000).

[7] J.-M. Frère, M. V. Libanov, and S. V. Troitsky, Phys. Lett. B479, 343 (2000).

[8] T. Banks, Nucl. Phys. B303, 172 (1988).

[9] E. Ma, Phys. Rev. D39, 1922 (1989).

[10] W. A. Bardeen, C. T. Hill, and M. Lindner, Phys. Rev. D41, 1647 (1990).

[11] M. Carena, T. E. Clark, C. E. M. Wagner, W. A. Bardeen, and K. Sasaki, Nucl. Phys. B369, 33 (1992).

[12] D. A. Demir and E. Ma, Phys. Rev. D62, 111901(R) (2000). 